\title{
Primary malignant fibrous histiocytoma of the liver
}

\author{
Georgios P. Fragulidis, Andreas Polydorou
}

Second Department of Surgery, “Aretaieio” Hospital, National and Kapodistrian University of Athens, School of Medicine, Athens, Greece Correspondence to: Georgios P. Fragulidis, MD, PhD. Second Department of Surgery, "Aretaieio" Hospital, National and Kapodistrian University of Athens, School of Medicine, 76, Vas.Sophias Ave.,11528, Athens, Attica, Greece. Email: gfragulidis@aretaieio.uoa.gr or gfragoulid@med.uoa.gr.

Submitted Aug 16, 2016. Accepted for publication Sep 05, 2016.

doi: $10.21037 / \mathrm{hbsn} .2016 .09 .05$

View this article at: http://dx.doi.org/10.21037/hbsn.2016.09.05
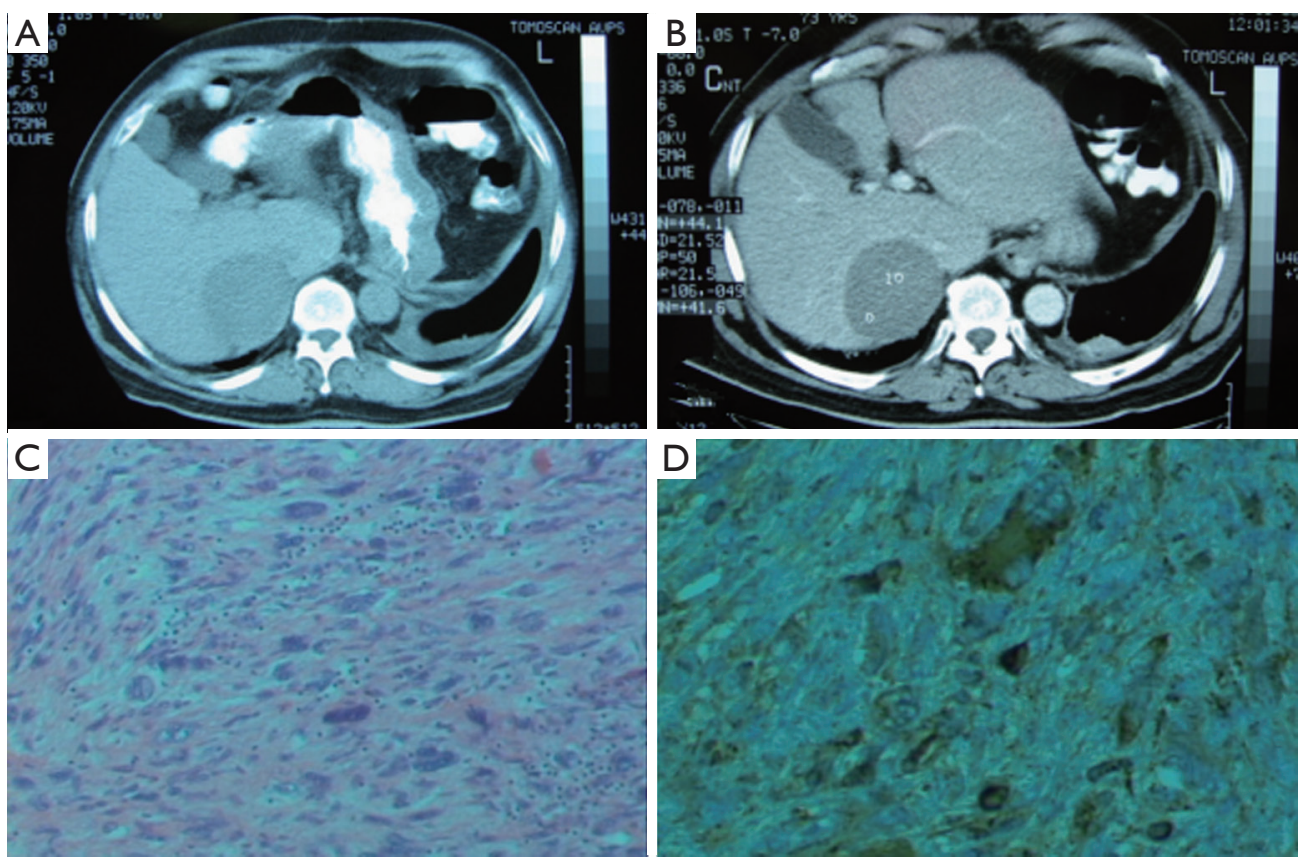

A 73-year-old male patient with an incidental finding of a solitary mass of the right lobe of the liver was referred to our hospital. Tumor serum markers were within normal limits. The CT scan showed a 7 -cm well circumscribed hypodense mass of liver segment VII enhanced minimally during the portal venous phase with marked enhancement on the delayed images (Panels A,B). Upper and lower GI endoscopy revealed negative findings. A liver resection of segments VII and VIII was performed. Histopathology revealed a circumscribed liver tumor measured $6.5 \mathrm{~cm} \times 8.5 \mathrm{~cm} \times 5 \mathrm{~cm}$ with a whiteyellowish sectional surface and firm consistency with a predominant spindle-shaped morphology and a mitotic activity of 1-3 mitoses/10 HPF. Immunohistaining presented a positive reaction of neoplastic cells to vimentin, alph-1antichemothrypsin and CD68 and a negative reaction to desmine and actine (Panels C,D, $\times 250$ ). The diagnosis of primary malignant fibrous histiocytoma was made according to morphology and the immunohistochemistry criteria.

\section{Acknowledgements}

None. 


\section{Footnote}

Conflicts of Interest: The authors have no conflicts of interest to declare.

Informed Consent: Written informed consent was obtained from the patient for publication of this manuscript and any accompanying images.

Cite this article as: Fragulidis GP, Polydorou A. Primary malignant fibrous histiocytoma of the liver. HepatoBiliary Surg Nutr 2016;5(5):449-450. doi: 10.21037/hbsn.2016.09.05 